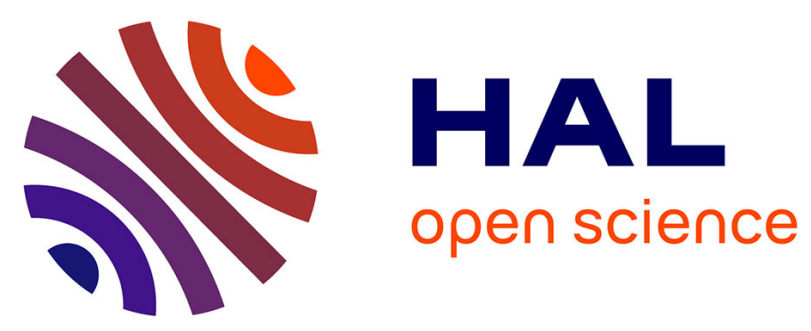

\title{
Control of shielding effectiveness of optically transparent films by modification of the edge termination geometry
}

Mathieu Croizer, Quentin Tricas, Philippe Besnier, Xavier Castel, Patrice

Foutrel

\section{- To cite this version:}

Mathieu Croizer, Quentin Tricas, Philippe Besnier, Xavier Castel, Patrice Foutrel. Control of shielding effectiveness of optically transparent films by modification of the edge termination geometry. IEEE Transactions on Electromagnetic Compatibility, 2020, 62 (6), pp.2431-2440. 10.1109/TEMC.2020.2982644 . hal-02554652

\section{HAL Id: hal-02554652 \\ https://hal.science/hal-02554652}

Submitted on 19 May 2020

HAL is a multi-disciplinary open access archive for the deposit and dissemination of scientific research documents, whether they are published or not. The documents may come from teaching and research institutions in France or abroad, or from public or private research centers.
L'archive ouverte pluridisciplinaire HAL, est destinée au dépôt et à la diffusion de documents scientifiques de niveau recherche, publiés ou non, émanant des établissements d'enseignement et de recherche français ou étrangers, des laboratoires publics ou privés. 


\title{
Control of Shielding Effectiveness of Optically Transparent Films by Modification of the Edge Termination Geometry
}

\author{
Mathieu Croizer, Quentin Tricas ，Philippe Besnier ，Senior Member, IEEE, Xavier Castel ， and Patrice Foutrel
}

\begin{abstract}
This article deals with the design, fabrication, and characterization of optically transparent electromagnetic screens to protect optical sensors against high-intensity radiated fields. To ensure the best tradeoff between high optical transparency over the entire visible light spectrum and high shielding effectiveness at microwaves, micrometric mesh metal films printed on glass substrates were selected. Changing the micrometric mesh pattern allows reaching various shielding effectiveness values required for different applications, but implies important adjustments of the design and fabrication processes. The variation of the number of contact ribbons between the mesh screen and its direct peripheral area consists of an alternative solution to modify the screen shielding effectiveness, while keeping the meshed part of the screen identical. An analytical model, which predicts the shielding effectiveness variation measured under statistically uniform illumination in a reverberation chamber as a function of the number of metal ribbons and the aperture sizes between them was specifically developed. Experimental results follow the trend predicted by the analytical model. As a result, adjusting the number of peripheral contact ribbons enables the shielding effectiveness to be fitted to specified requirements at constant optical transparency.
\end{abstract}

Index Terms-Electromagnetic shielding, high-intensity radiated fields (HIRFs), micrometric mesh metal film, optical transparency, reverberation chamber, shielding effectiveness.

\section{INTRODUCTION}

$\mathbf{N}$ UMEROUS electronic systems use optical sensors for various fields of application on sea, land, and in the air. In a lot of cases, the embedded electronic are very sensitive

Manuscript received October 10, 2019; revised February 10, 2020; accepted March 2, 2020. This work was supported in part by SAFRAN Electronics \& Defense under Grant 142120, in part by the European Union through the European Regional Development Fund, in part by the Ministry of Higher Education and Research, in part by the Région Bretagne, and in part by the Département des Côtes d'Armor and Saint-Brieuc Armor Agglomération, through the CPER Projects 2015-2020 MATECOM and SOPHIE/STIC \& Ondes. (Corresponding author: Quentin Tricas.)

Mathieu Croizer, Philippe Besnier, and Xavier Castel are with the Univ Rennes, INSA Rennes, CNRS, IETR - UMR 6164, F-35000, Rennes, France (e-mail: mathieu.croizer@univ-rennes1.fr; philippe.besnier@insa-rennes.fr; xavier.castel@univ-rennes1.fr).

Quentin Tricas is with the Univ Rennes, INSA Rennes, CNRS, IETR - UMR 6164, F-35000, Rennes, France and also with SAFRAN Electronics \& Defense, F-95610 Eragny-sur-Oise, France (e-mail: quentin.tricas@univ-rennes1.fr).

Patrice Foutrel is with the SAFRAN Electronics \& Defense, F-95610 Eragnysur-Oise, France (e-mail: patrice.foutrel@ safrangroup.com).

Color versions of one or more of the figures in this article are available online at http://ieeexplore.ieee.org.

Digital Object Identifier 10.1109/TEMC.2020.2982644 to high-intensity radiated fields (HIRFs) aggression and need to be protected. Some guidelines have been given by various official bodies, such as the European Aviation Safety Agency or the Federal Aviation Administration (FAA) for the aviation sector in the USA. Typical electric field levels requirements are provided in the FAA advisory circular AC 20-158A, but more stringent levels may be required for specific applications [1]. In the open literature, many studies explored and tested different routes to protect aircraft systems against HIRFs. Among the proposed solutions, lightweight shielding with a high level of optical transparency over the entire visible light spectrum, such as transparent and conducting films printed on glass substrates is particularly discussed. Among the available transparent and conducting oxide (TCO) materials, namely indium tin oxide [2], Al-doped zinc oxide [3], ultrathin metal film [4], or/and $\mathrm{TCO} /$ metal multilayers [2], the most relevant solution consists of a micrometric mesh metal film whose apertures are larger than the metal strip width, and smaller than the minimum operating wavelength of the aggressor beam [5]. With this solution, there is almost no correlation between the optical transparency and the conducting layer thickness, whereas it is a critical parameter for solution based on continuous conducting films. In a recent paper [6], a transmission line model to determine simultaneously the optical transparency $T$, the shielding effectiveness (SE), and the sheet resistance $R_{S}$ of such films was developed and validated.

Nevertheless, SE performance requirements are different from one sensor to another, because they depend on operational conditions and electromagnetic compatibility requirements for embedded electronics. In some cases, high SE protection may be unnecessary, and even induces unwanted high Q-factor within the protected area. It is therefore important to find cost-effective technical solutions to modulate the SE level of the screens. A direct route consists of designing the micrometric mesh metal screen specifically for each application, i.e., computing the mesh parameters (the pitch $g$ and the strip width $2 a$ ) as in [6] toward the targeted SE. Another route is to provide a controllable variation of the equivalent contact impedance $\left(Z_{\mathrm{Co}}\right)$ between a predetermined micrometric mesh metal film and its direct peripheral area. The impact of $Z_{\mathrm{Co}}$ on SE has been extensively studied in the literature [7]-[12]. Electromagnetic gaskets provide low resistance $R_{\mathrm{Co}}$, but SE may depend on their compression level [7], [8]. The geometry of the gaskets and the materials they are made of also affect the resistance value, and are critical for the SE 
control [9]-[11]. In [12], Catrysse et al. inserted pieces of paper with various dimensions between the shielding material sample and its surrounding support to increase the overall resistance. They experimentally demonstrated the possibility of varying the SE by varying the discontinuities distribution between the sample and its support. Nevertheless, while the trends of the SE were well predicted, quantifying SE variations was complex. The electromagnetic screen design we present here is based on the control of the contact impedance $Z_{\mathrm{Co}}$ between a fixed mesh metal film and its direct peripheral area, by using a variable number $N$ of peripheral metal ribbons to connect them, and thus modifying the size of the resulting peripheral apertures. This design offers a way to considerably modify the SE of the resulting screens, without change of their central area. This allows for the production of shieldings with very diverse SE levels, while only slightly modifying their pattern. This approach could be of interest to facilitate industrial scaling, simplify production, and save costs. It also ensures that the optical transparency of the central area is identical, regardless of the desired SE, which is not the case when the geometrical parameters $g$ and $2 a$ of the mesh have to be modified to adjust the SE level.

Accordingly, the article is organized as follows. Section II depicts the proposed solution. Section III develops the theoretical model of SE variation based on an effective area aperture assessment. Fabrication process of the shielding screen samples is detailed in Section IV. In Section V, the shielding experimental setup is presented and experimental validation is carried out in the $2-34 \mathrm{GHz}$ frequency range. Finally, conclusions are drawn in Section VI.

\section{DESCRIPTION OF THE SOLUTION}

To control the SE exhibited by a screen, our proposal is based on the creation of a controlled connection area at the periphery of the shielding screen, while maintaining the contact impedance with the external support to be as low as possible. The connection area is made of a series of metal ribbons connecting the edges of the micrometric mesh metal area and a metal square ring at the periphery of the sample (see Fig. 1). The square ring itself ensures an excellent electrical contact with the external support. In that case, apertures between the low impedance metal ribbons play the role of the contact discontinuities that were realized by pieces of paper in [12]. As a result, SE of the screen is controlled by the number and the size of the apertures, as a function of the number of metal ribbons. This article is therefore dedicated to the development of a specific theoretical but approximate model designed to quantify the impact of the number of contact ribbons on SE, and to the comparison of the theoretical results with those from SE measurements.

\section{THEORETICAL MODEL}

In this section, the specific analytical model is introduced to evaluate the SE response of a micrometric mesh metal area surrounded by metal ribbons (see Fig. 1). The two extreme responses of the model are described as follows: the highest $\mathrm{SE}$ is achieved by the full micrometric mesh metal sample [see Fig. 2(b)], while the lowest SE is achieved by the micrometric

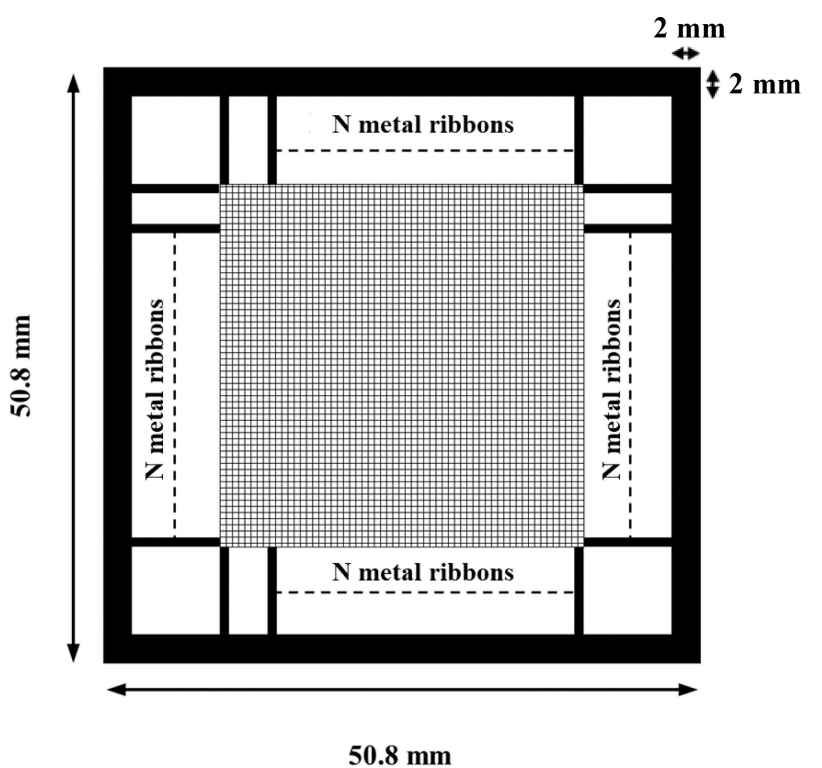

Fig. 1. Top view of the micrometric mesh metal area surrounded by $4 \times N$ metal ribbons connected to a square metal ring.

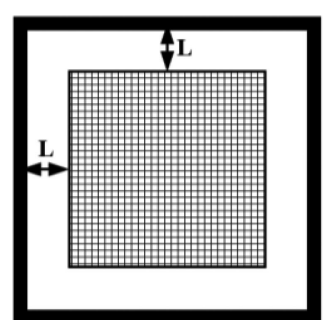

(a)

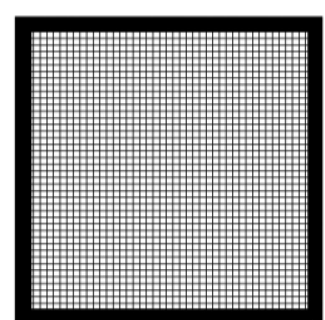

(b)
Fig. 2. (a) Micrometric mesh metal area surrounded by an open area, and (b) full micrometric mesh metal sample.

mesh metal area surrounded by an open area [see Fig. 2(a)]. In the first extreme case, the complex impedance $Z_{\mathrm{mf}}$ of the micrometric mesh metal film is computed from (1) derived in [6] as follows:

$$
\begin{aligned}
Z_{\mathrm{mf}}= & \frac{1+j}{\sigma_{m} \delta_{m} \tanh \left((1+j) t_{m} / \delta_{m}\right)} \times \frac{g}{2 a} \\
& +j Z_{0} \frac{g}{\lambda_{0}} \ln \left(\frac{1}{\sin (\pi a / g)}\right)
\end{aligned}
$$

where $\sigma_{m}$ is the conductivity of the metal, $\delta_{m}$ is the skin depth at the operating frequency, $t_{m}$ is the thickness of the mesh metal screen, $g$ is the mesh pitch, $2 a$ is the metal strip width of the mesh, $Z_{0}$ is the wave impedance of free space, and $\lambda_{0}$ is the wavelength at the operating frequency. In the second extreme case, the spacing $L$ of the open area remains narrow with respect to the minimum wavelength for which the SE performance is expected. We select a spacing strictly lower than $\lambda_{0} / 4$ at $20 \mathrm{GHz}$ $(L=3.4 \mathrm{~mm}$ ). Above $20 \mathrm{GHz}$, no significant modulation of SE is expected, as the value of the wavelength of the electromagnetic waves starts to be too close to (or even, higher than) $L$. Between these two extreme cases, micrometric mesh 


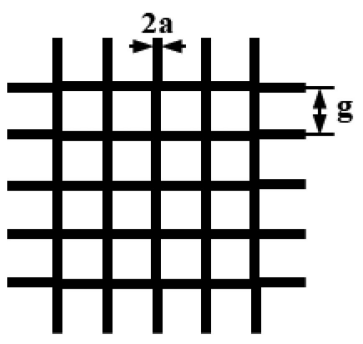

(a)

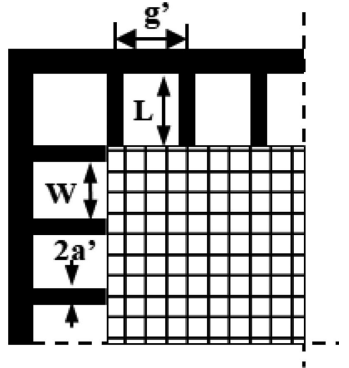

(b)

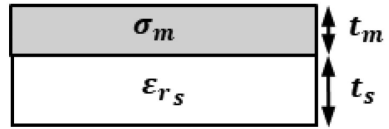

(c)

Fig. 3. (a) Top view of a square micrometric mesh metal film. $2 a$ is the metal strip width and $g$ is the mesh pitch. (b) Top view detail of the square micrometric mesh metal film surrounded by the metal ribbons. $2 a^{\prime}$ is the ribbon width; $g$ ' is the ribbon pitch; $L$ is the ribbon length, and $W$ is the aperture width between two successive ribbons. (c) Cross-sectional view of $t_{m}$-thick mesh metal layer printed on $t_{s}$-thick substrate. $\sigma_{m}$ is the layer conductivity and $\varepsilon_{r s}$ is the substrate permittivity.

metal area surrounded by metal ribbons is investigated. The corresponding SE model combines the SE response of the full micrometric mesh metal film as defined in [6] with the effective area associated with the polarizabilities of the set of apertures due to the peripheral metal ribbons. Moreover, the metal ribbon contact impedance $\left(Z_{\mathrm{Co}}\right)$ should remain at the same order of magnitude (or even lower) than the surface impedance of the micrometric mesh metal film $\left(Z_{\mathrm{mf}}\right)$.

\section{A. SE Determination}

SE calculation has already been carried out in [6] for continuous and mesh conducting layers stacked onto a transparent substrate. In this article, we restrict the study to a single micrometric mesh metal film printed on a lossless glass substrate. In [6], the SE estimation of the mesh metal sample was based on a transmission line model under plane wave illumination rather than under random plane wave spectrum illumination, such as met in reverberation chambers. In this article, the random plane wave spectrum illumination (random polarization and random angle of incidence) is used to determine the SE of the combined mesh metal film and peripheral ribbons network. Under this hypothesis, the effective area of an unloaded or loaded aperture is half that of the same apertures under plane wave illumination [13]. Based on the new configuration (see Fig. 3), the SE provided by such samples depends on both the mesh metal film characteristics and the electromagnetic behavior of the peripheral aperture array. Therefore, total SE depends on the combination of their respective impedances or effective areas. As far as the peripheral array of apertures is concerned, it also depends on the field polarization.

First, we determine the effective area of the aperture array built by the peripheral metal ribbons. In [14], Bethe calculated the

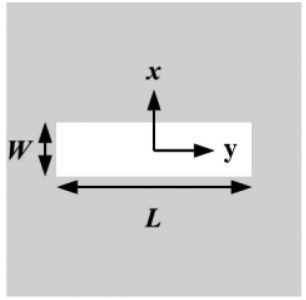

(a)

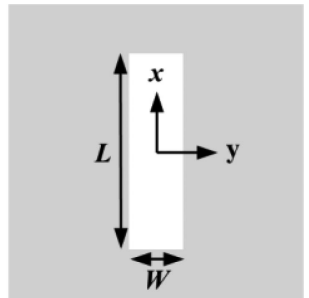

(b)
Fig. 4. (a) Details of a $W \times L$ aperture in a thin metal film illuminated by a plane TE wave with the aperture length $L$ aligned in $y$-direction; (b) aligned in $x$-direction.

diffracted fields by small holes through the electric and magnetic dipolar moments $(\vec{P}, \vec{M})$ expressed as follows:

$$
\left\{\begin{array}{l}
\vec{P}=\dot{\alpha}_{e} \varepsilon \overrightarrow{E_{\mathrm{inc}}} \\
\vec{M}=\dot{\alpha}_{m} \overrightarrow{H_{\mathrm{inc}}}
\end{array}\right.
$$

where $\varepsilon$ is the dielectric permittivity of the medium, $\overrightarrow{E_{\text {inc }}}$ is the normal component of the incident electric field, $\overrightarrow{H_{\mathrm{inc}}}$ is the tangential component of the incident magnetic field, and $\alpha_{e}$ and $\alpha_{m}$ are respectively the electric and magnetic polarizabilities of a small aperture in comparison with the operating wavelength. These polarizabilities are geometric factors based on the aperture shape and size. Bethe's theoretical study focused on circular apertures, but following studies expanded the theory to various shapes [15]-[17]. Approximate polynomial equations were also proposed in [18] and [19]. Given a set of rectangular apertures, an acceptable tradeoff consists in using elliptical apertures expression [16] and applying a normalization factor. The latter corresponds to the ratio of their area to the power of 3/2 [15, Figs. 100 and 101]. Apertures are illuminated by a set of plane waves with random polarization and incidence angle. We do not neglect the electric dipolar moment, though the scattered energy is mainly due to the magnetic field leakage [13]. The magnetic polarizability values depend on the aperture orientation compared to the incident plane wave polarization, as shown in Fig. 4. Therefore, the magnetic polarizabilities are defined by (3) as follows [16], [17]:

$$
\begin{aligned}
W \rightarrow x, L \rightarrow y \Rightarrow \alpha_{m_{1}} & =\frac{\pi L^{3} m}{6(K(\sqrt{m})-E(\sqrt{m}))} \\
W \rightarrow y, L \rightarrow x \Rightarrow \alpha_{m_{2}} & =\frac{\pi L^{3} m(1-m)}{6(E(\sqrt{m})-(1-m) K(\sqrt{m}))} \\
m & =1-\left(\frac{W}{L}\right)^{2}
\end{aligned}
$$

where $K$ and $E$ are the complete elliptic integrals of the first and second kind. The electric polarizability is defined as follows:

$$
\alpha_{e}=\frac{\pi L^{2} W}{6 E(\sqrt{m})} .
$$

Accounting for random polarization and incidence angle of the impinging waves, an average magnetic polarizability is 
defined as follows:

$$
\alpha_{m_{\text {average }}}=\frac{\alpha_{m_{1}}+\alpha_{m_{2}}}{2} .
$$

So far, the mutual coupling between apertures has been neglected. The coupling factor depends on the field polarization with respect to the alignment of the line array of the peripheral apertures. This was investigated from analytical developments in [20]. It appeared that contributions from the coupling effects of many neighbor circular apertures is nonnegligible. However, analytical approximate expressions were limited to distances of at least 1.5 times the radius of the circular aperture. From [21], numerical investigations suggest that coupling effects are limited if aperture spacing ( $\mathrm{sp}$ ) is greater than half of the aperture size, but may reach a significant level for sp equal to one-fifth of the aperture size. The aperture array surrounding the mesh metal film in Fig. 1 consists of millimeter-size apertures separated by ribbons of only 200- $\mu \mathrm{m}$ width. According to Jaggard [21], the polarizabilities of circular apertures are modified by their neighbors as follows:

$$
\frac{\alpha^{c}}{\alpha^{0}} \approx\left(1-\frac{\alpha^{0}}{4 \pi(\phi+\mathrm{sp})}\right)^{-1} \text { for } \mathrm{sp} \geq \frac{\phi}{2}
$$

where $\mathrm{sp}$ is the separation distance between two successive apertures and $\phi$ is their diameter; $\alpha^{0}$ is either the magnetic or the electric polarizability of an isolated aperture and $\alpha^{c}$ is the same quantity for coupled apertures. For very large sp, $\alpha^{0} \approx \alpha^{c}$ but the derivative of $\frac{\alpha^{c}}{\alpha^{0}}$ with respect to $\phi$ is proportional to $\phi^{2}$, $\alpha^{0}$ being proportional to $\phi^{3}$. Accordingly, the coupling factor evolves with the aperture surface. For very small sp, we assume that this trend remains identical. Therefore, it comes that an empirical estimation of $\alpha^{c}$ may be proposed as

$$
\frac{\alpha^{c}}{\alpha^{0}}=C_{0} \frac{L W}{L_{\min } W_{\min }} \text { for } \mathrm{sp} \ll \phi
$$

where $L_{\min } W_{\min }$ is taken as the smallest aperture size corresponding to the maximum number of ribbons of the samples (132 here). $C_{0}$ is a constant which was fit to the experimental SE obtained in $L_{\min } W_{\min }$ case. It was found to be $C_{0}=4.5$.

In the case of square apertures $(W=L=d)$, the magnetic polarizability approximated with a circular polarizability and the electric polarizability [15] are expressed as follows:

$$
\begin{aligned}
\alpha_{m_{\left(\frac{1}{2}\right)}} & =2 \times \frac{d^{3}}{3} \\
\alpha_{e} & =\frac{d^{3}}{3} .
\end{aligned}
$$

The application of the above mentioned normalization factor in [15] provides

$$
\alpha_{\text {norm }} \approx\left(\frac{4}{\pi}\right)^{3 / 2} \alpha
$$

the term $\frac{4}{\pi}$ being the value of the rectangular (square) aperture area divided by the elliptical (circular) aperture area. In (6), $\alpha$ stands for any electric or magnetic polarizability. The normalization and coupling factors are applied to compute the square/rectangular aperture magnetic polarizability as follows:

$$
\begin{aligned}
\alpha_{m\left(\frac{1}{2}\right)}^{\prime} & \approx\left(\frac{4}{\pi}\right)^{3 / 2} \times \frac{\alpha^{c}}{\alpha^{0}} \alpha_{m}{ }_{\left(\frac{1}{2}\right)} \\
\alpha_{e}^{\prime} & \approx\left(\frac{4}{\pi}\right)^{3 / 2} \times \frac{\alpha^{c}}{\alpha^{0}} \alpha_{e}
\end{aligned}
$$

$\frac{4}{\pi}$ being the value of the square/rectangular aperture area divided by the circular/elliptical aperture area.

Accordingly, a transmission coefficient $\tau$ can be deduced from the ratio between the transmitted and incident energy density through an aperture in the far field as a function of its surface area $A$ as follows [13], [21]:

$$
\tau=\frac{4 \pi^{3}}{9 \times A \times \lambda_{0}^{4}}\left(2 \alpha_{m \text { average }}^{\prime 2}+\alpha_{e}^{\prime 2}\right) .
$$

The total effective area of the set of apertures $\left(A_{\mathrm{eff}_{\mathrm{ap}}^{T}}\right)$ is equal to the summation of all effective aperture areas. Nevertheless, (11) is only valid if apertures are small in comparison with the operating wavelength. Depending on the number of ribbon connections, this condition is not systematically fulfilled. As a rough approximation, the effective area of an aperture is limited by half of its physical area. Therefore, the total effective area of the set of apertures is computed as follows:

$$
A_{\mathrm{eff}_{\mathrm{ap}}^{T}}^{T}=\sum_{i=1}^{N_{a p}} \min \left(\tau_{i} A_{\mathrm{ap}_{i}}, A_{\mathrm{ap}_{i}} / 2\right) .
$$

To account for the residual SE associated with the substrate permittivity, according to its thickness, the effective area calculated in (12) must be corrected. This correction factor is provided from the calculation of the $S_{21}$ parameter through the substrate [6, eq.(8)]. Finally, the modified effective area of the peripheral apertures is given by

$$
A_{\mathrm{eff}_{\mathrm{ap}}^{\mathrm{TS}}}^{\mathrm{TS}}=A_{\mathrm{eff}_{\mathrm{ap}}^{T}}^{T}\left(1-S_{21}^{2}\right) .
$$

Once the effective area of apertures assessed, the effective surface area of the mesh metal film $A_{\text {eff mf }}$ needs to be introduced. It is related to $\mathrm{SE}_{\mathrm{mf}}$ of the full mesh sample as calculated from [6], and to the half-surface area $A_{\mathrm{mf}} / 2$ under random plane wave illumination as follows:

$$
A_{\mathrm{eff} \mathrm{mf}}=\left(A_{\mathrm{mf}} / 2\right) S E_{\mathrm{mf}} .
$$

Consequently, SE of the studied samples is estimated as follows:

$$
\mathrm{SE}=\frac{A_{\mathrm{eff}_{\mathrm{ap}}^{\mathrm{TS}}}+A_{\mathrm{eff} \mathrm{mf}}}{\left(A_{\text {total }} / 2\right)}
$$

where $A_{\text {total }}$ is the total area of the sample. The SE is therefore plotted in $\left(-10 \log _{10}(\mathrm{SE})\right)$.

\section{B. $\mathrm{Z}_{\text {Co }}$ Assessment}

The metal ribbon contact impedance $Z_{\mathrm{Co}}$ depends on the ribbon geometric parameters: its length $L$, its width $2 a$, its 
TABLE I

GEOMETRIC SAMPLE PARAMETERS

\begin{tabular}{cc}
\hline \hline $2 a$ & $10 \mu \mathrm{m}$ \\
$g$ & $200 \mu \mathrm{m}$ \\
$\begin{array}{c}\text { Number of metal } \\
\text { ribbons }\end{array}$ & {$[132 ; 92 ; 44 ; 24]$} \\
$2 a^{\prime}$ & $200 \mu \mathrm{m}$ \\
$g^{\prime}$ & {$[1.24 ; 1.81 ; 3.98 ; 7.96] \mathrm{mm}$} \\
$L$ & $3.4 \mathrm{~mm}$ \\
$W=g^{\prime}-2 a^{\prime}$ & {$[1.04 ; 1.61 ; 3.78 ; 7.76] \mathrm{mm}$}
\end{tabular}

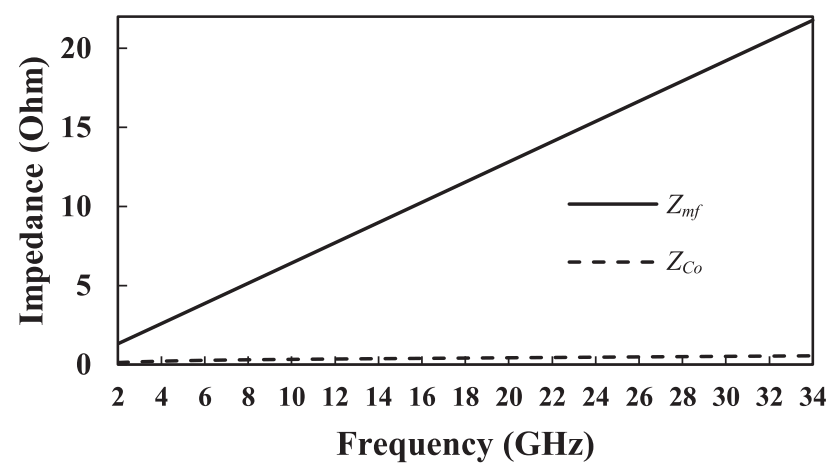

Fig. 5. Variation of the mesh metal film and metal ribbon contact impedances versus frequency.

thickness $t_{m}\left(t_{m} \ll 2 a^{\prime}\right)$ as follows [22]:

$$
Z_{\mathrm{Co}}=\frac{Z_{s}}{2 j} \tan \left(\frac{(1-j) t_{m}}{2 \delta_{m}}\right) \frac{L}{2 a^{\prime}}
$$

where $Z_{s}$ is the surface impedance of the metal ribbon given by (17) as follows:

$$
Z_{s}=\frac{1+j}{\sigma_{m} \delta_{m}}
$$

\section{Analytical Model}

The parameters of the studied samples (see Fig. 3) are summarized in Table I. The dimensions of the glass substrate are $50.8 \mathrm{~mm} \times 50.8 \mathrm{~mm} \times 0.7 \mathrm{~mm}$. Its relative permittivity is $\varepsilon_{\mathrm{r}}=5.0$ with negligible loss at the operating frequencies. A centered $40 \mathrm{~mm} \times 40 \mathrm{~mm}$ micrometric mesh metal film is printed onto the glass substrate. We use the conductivity value of the pure metal silver at $20^{\circ} \mathrm{C}$ (the best electrical conductor of the periodic table with $\sigma_{m}=6.3 \times 10^{7} \mathrm{~S} / \mathrm{m}$ [23]) with a metal thickness $t_{m}=2 \mu \mathrm{m}$. With these parameters and according to (1) and (16), 200- $\mu \mathrm{m}$-width silver ribbons are sufficient to ensure $Z_{\mathrm{Co}}$ lower than $Z_{\mathrm{mf}}$ over the entire operating frequency range (see Fig. 5).

The SE is computed from an analytical model as described in Section III-A. This model requires $\mathrm{SE}_{\mathrm{mf}}$ of the full micrometric mesh silver film as computed in [6]. Fig. 6 highlights a significant variation of SE with respect to the number of silver ribbons

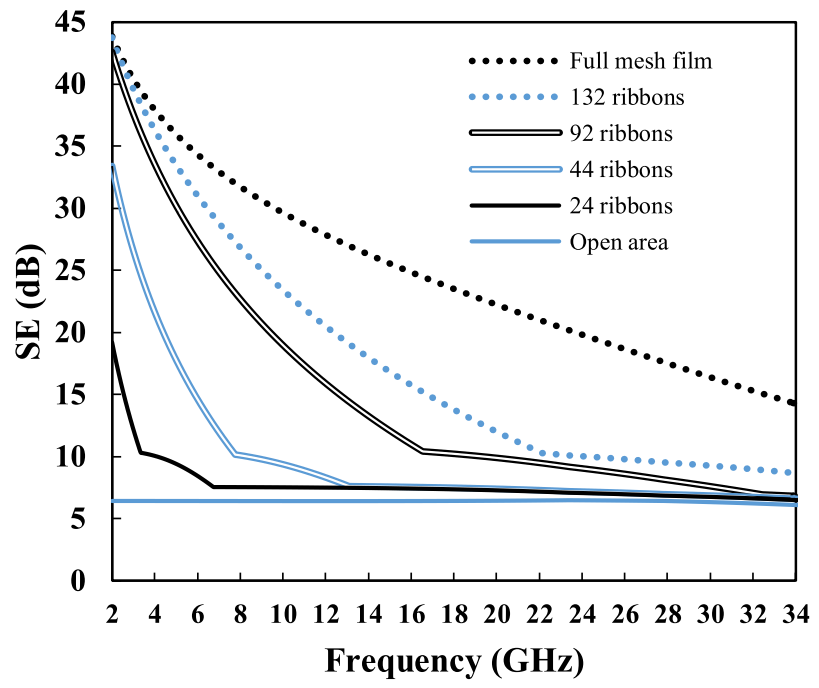

Fig. 6. SE analytical model for various numbers of silver ribbons connected to the micrometric mesh metal film.

connected to the mesh silver screen. As expected, the highest SE performance is achieved with the full mesh silver film, and is gradually altered with the decrease in the number of silver ribbon connections. Nevertheless, beyond $25 \mathrm{GHz}$, the magnitude of the SE goes flat no matter what the number of silver connections is. The SE minimal level $(\sim 7 \mathrm{~dB})$ is controlled by the air/glass permittivity contrast, as the size of the aperture between two consecutive silver ribbons is significantly larger than the operating wavelength value $\lambda_{0}$. As expected, as the number of metal connections decreases, SE minimal level is reached at lower frequencies. SE tends to reach the minimal plateau at frequencies close to 7 and $22 \mathrm{GHz}$ for the 24 and 132 ribbons samples, respectively. For both samples, these frequencies correspond to roughly quarter-wavelength rectangular apertures whose length is $g^{\prime}=7.97 \mathrm{~mm}$ (24 ribbons sample) and $L=3.4 \mathrm{~mm}$ (132 ribbons sample).

\section{FABRICATION PROCESS}

\section{A. Metal Deposition}

Samples consist of two metal layers stacked on a $50.8 \mathrm{~mm} \times 50.8 \mathrm{~mm} \times 0.7 \mathrm{~mm}$ soda-lime glass substrate: a 2 - $\mu \mathrm{m}$-thick silver layer onto a 5 -nm-thick titanium underlayer deposited by radiofrequency magnetron sputtering at room temperature. The titanium underlayer is used here only to ensure the strong adhesion of the silver overlayer onto the glass substrate.

The sputtering chamber contains two sputtering targets (100 $\mathrm{mm}$ in diameter): a silver disc $(99.999 \%$ purity) and a titanium disc $(99.995 \%$ purity). This configuration makes it possible to grow a single layer or a multilayer in a single run without breaking vacuum. The glass substrate clamped onto the sample holder revolves around a central axis, which allows positioning the substrate in front of the selected target. A RF power density of $25 \times 10^{-3} \mathrm{~W} / \mathrm{mm}^{2}$ is used on each working target; the argon working pressure is $1.45 \mathrm{~Pa}$, and the deposition time is adjusted to achieve the desired metal thickness. The 


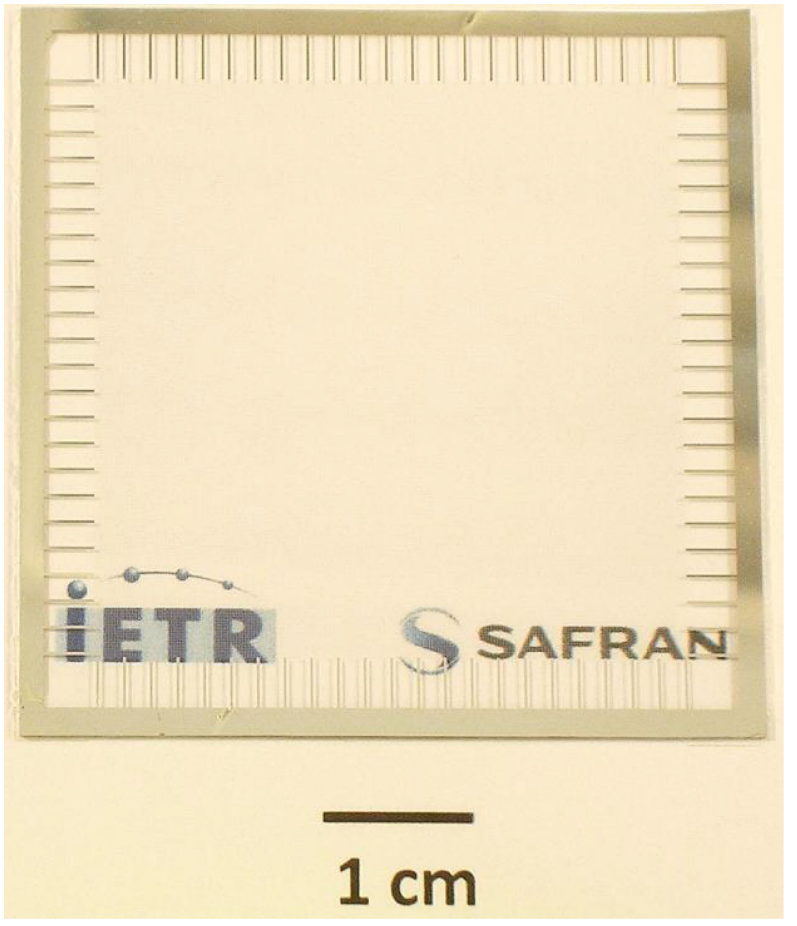

Fig. 7. Picture of the micrometric mesh silver/titanium film with 92 peripheral connected silver/titanium ribbons printed onto a $50.8 \mathrm{~mm} \times 50.8 \mathrm{~mm} \times 0.7 \mathrm{~mm}$ soda-lime glass substrate. Sample is placed above the companies logos.

sputtering rate has been calibrated for each target and is equal to $137 \mathrm{~nm} / \mathrm{min}$ for silver (Ag) and $20 \mathrm{~nm} / \mathrm{min}$ for titanium (Ti). The total thickness of the sample is therefore controlled through sputtering time and checked afterward by stylus profilometry.

\section{B. Square Mesh and Ribbon Patterning}

To complete the fabrication of the square micrometric mesh silver/titanium samples with various numbers of ribbons, additional stages are required. After the metallization step, the samples are spin-coated with a photosensitive resin layer. Then, they are exposed to UV light through specific photomasks with the selected pattern. After developing the exposed photoresist, $\mathrm{Ag}$ and $\mathrm{Ti}$ are ready to be engraved in suitable chemical etching solutions at room temperature. The silver film is etched in a $\mathrm{HNO}_{3} / \mathrm{H}_{3} \mathrm{PO}_{4} / \mathrm{CH}_{3} \mathrm{COOH} / \mathrm{H}_{2} \mathrm{O}$ solution with a volume ratio of 10:40:40:10 and the titanium film in a $\mathrm{HF} / \mathrm{H}_{2} \mathrm{O}$ solution with a volume ratio of 3:97. Stripping of the photoresist leaves the samples with a centered periodic array of square micrometric apertures connected to peripheral silver ribbons. Fig. 7 shows the micrometric mesh silver/titanium film with 92 peripheral connected silver/titanium ribbons printed onto a soda-lime glass substrate.

\section{EXPERIMENTAL SETUP AND RESUlts}

\section{A. SE Measurements}

SE measurements are based on the nested reverberation chamber procedure as described in [6] and originally proposed in [24]. A homemade Faraday cage $(580 \mathrm{~mm} \times 590 \mathrm{~mm}$

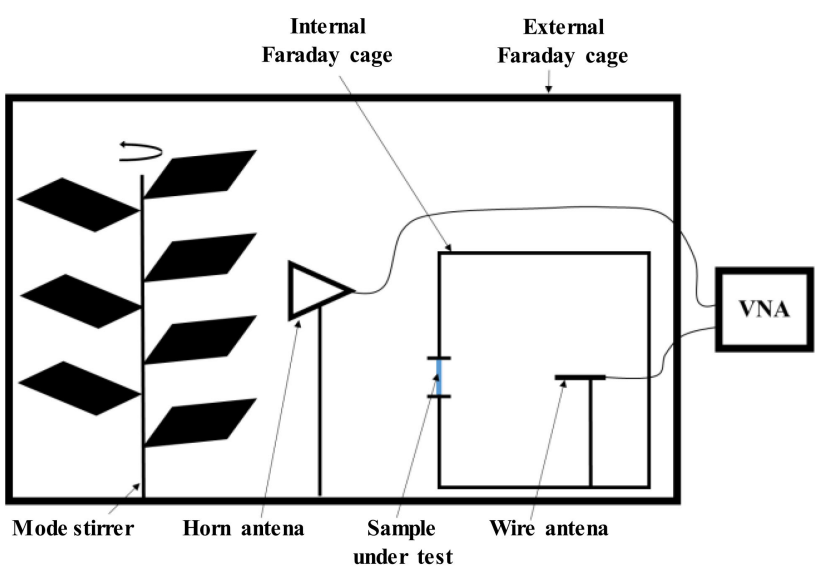

(a)

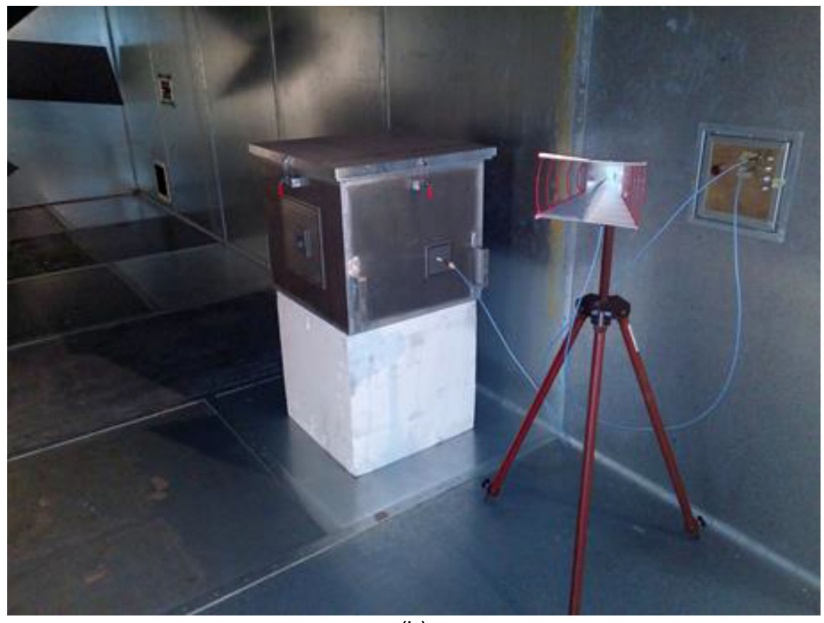

(b)

Fig. 8. Measurement setup used to determine sample SE variation versus frequency as described in [6]. (a) Schematic view and (b) picture of the setup.

$\times 600 \mathrm{~mm})$ is placed inside a larger reverberation chamber $(2.9 \mathrm{~m} \times 3.7 \mathrm{~m} \times 8.7 \mathrm{~m})$, as shown in Fig. 8. The Faraday cage was fabricated from 10 -mm-thick AlCuMg 1 metal plates. Measurements are carried out in the $2-34 \mathrm{GHz}$ frequency band from a four ports Rhode \& Schwarz Vector Network Analyzer (VNA). The procedure called $\mathrm{SE}_{3}$ described in [24, refer to (12)] is used. On the one hand, the modification of the Q-factor of the Faraday cage, which occurs when the aperture is first unloaded, and then loaded by the samples under test, is compensated. On the other hand, the Q-factor of the larger reverberation chamber is not affected by this change. To remove the statistical fluctuations from SE measurements, the corresponding factors are neglected. Therefore, two antennas only are connected to the VNA, as depicted in [6]. Two different transmitting horn antennas (2-18 GHz and 18-34 GHz) are successively used to supply the electromagnetic field into the reverberation chamber. The receiving antenna is a 15-cm-long wire placed into the Faraday cage. The results obtained with the wire antenna are equivalent to those that would have been obtained with a broadband horn antenna in the hypothesis of a diffuse field environment within the Faraday cage. 
TABLE II

VNA PARAMETERS AND STIRRING POSITIONS

\begin{tabular}{ccc}
\hline \hline & $2-18 \mathrm{GHz}$ & $18-34 \mathrm{GHz}$ \\
\hline Power & $10 \mathrm{dBm}$ & $13 \mathrm{dBm}$ \\
$\begin{array}{c}\text { Resolution bandwidth } \\
\text { filter }\end{array}$ & $500 \mathrm{~Hz}$ \\
Number of points & 20001 \\
$\begin{array}{c}\text { Frequency averaging } \\
\text { bandwidth } \\
\begin{array}{c}\text { Number of stirring } \\
\text { positions }\end{array}\end{array}$ & $96 \mathrm{MHz}$ \\
\hline \hline
\end{tabular}

A 210-mm-side-length square window is made in one side of the Faraday cage. Therefore, the maximum sample dimensions cannot exceed $200 \mathrm{~mm} \times 200 \mathrm{~mm}$. A specific sample holder has been machined to test the $\sim 50$-mm-side-length samples resulting in a 46.8-mm-side-length-square open window. Galvanic contact between the Faraday cage and the samples under test is ensured by uniformly spread screws, linking the sample holder to the wall of the Faraday cage.

In the $2-34 \mathrm{GHz}$ frequency range, the reverberation chamber generates an ideal random field, which is statistically uniform in the chamber volume. This ideal random field is theoretically equivalent to the sum of many plane waves with random, uniformly spread phase, incidence and polarization angles. The aperture of the inner Faraday cage is illuminated by such a random field over a half-sphere. In these conditions, two measurements are required with an unloaded and a loaded aperture to estimate the SE of the sample under test. Therefore, the experimental results are directly comparable to those retrieved from the SE analytical model as described in Section III-A.

The dynamic range of experimental SE measurements is assessed from the measurement of two extreme configurations: an unloaded cage aperture and a cage aperture closed by a 6-mm-thick piece of $\mathrm{AlCuMg} 1$ metal, considered as a nearly perfect shielding screen. All measurements presented in the following section lie within the limits of the dynamic range with the setup parameters reported in Table II.

\section{B. Sheet Resistance $\left(R_{S}\right)$ and Optical Transparency $(T)$ Measurements}

The sheet resistance $R_{S}$ was measured by a standard four probe setup. It consists of four equally spaced inline metal probes in contact at the center of the sample under test. A stabilized current is fed through the two outer probes. A microvoltmeter measures the voltage across the two inner probes to compute $R_{S}$.

After air (blank) calibration, the optical transparency of the samples was recorded at normal incidence in the 200-1100 nm spectral range by a UV-visible spectrophotometer. It is worth noting that the measured optical transparency includes the Fresnel loss of the soda-lime glass substrate ( $4 \%$ per side).

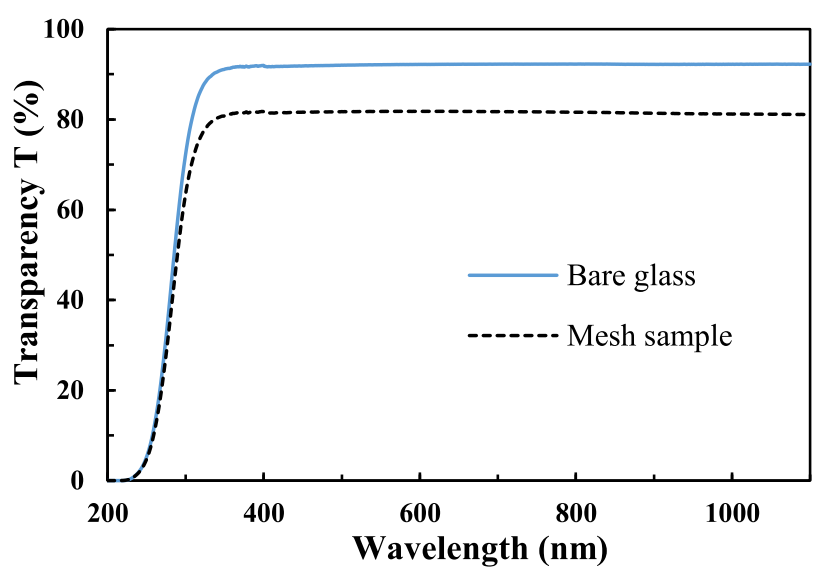

Fig. 9. Measured optical transparency of the micrometric mesh central part of the samples with $g=200 \mu \mathrm{m}$ and $2 a=10 \mu \mathrm{m}$. Spectrum of the bare soda-lime glass is also plotted for comparison.

\section{Experimental Results}

The measured sheet resistance of all samples is equal to $0.20 \Omega / \mathrm{sq}$, which corresponds to the theoretical one $(0.16 \Omega / \mathrm{sq})$ computed from the mesh parameters as specified in Table I as follows [25]:

$$
R_{S}=\frac{g}{2 a} \times \frac{1}{\sigma_{m} t_{m}} .
$$

The dc resistance of the silver ribbons is small compared with the sheet resistance value of the mesh metal film. Moreover, $R_{S}$ value is equal to the real part of the complex impedance $Z_{\mathrm{mf}}$ of the micrometric mesh silver film in dc mode [see (1)].

The measured optical transparency of the mesh metal film is $T \approx 81 \%$ over the entire visible light spectrum (see Fig. 9) in full accordance with the theoretical value $(T=83.1 \%)$ as follows [6]-[25]:

$$
T=\left(\frac{g-2 a}{g}\right)^{2} \times T_{\text {sub }}
$$

where $T_{\text {sub }}=92 \%$ is the optical transparency of the bare sodalime glass substrate (see Fig. 9).

SE measurements of the transparent samples with various numbers of silver ribbons (see Figs. 10-13) are compared with the numerical results computed from the analytical model described in Section III-A. At low frequency, the responses of the sample with 132 and 92 silver ribbons are altered by fluctuations due to the limited size of the sample. SE performance of the 132, 92, and 44 silver ribbons samples (see Figs. 10-12) fit to the theoretical results. The results of the 24 silver ribbons sample (see Fig. 13) highlight a numerical underestimation of the cutoff frequency, above which SE value decreases sharply. However, Figs. 6 and 14 provide the expected trend and dynamic range of SE variations, which is the main purpose. On the one hand, the significant SE change is observed both in theory and experiment in the $2-25-\mathrm{GHz}$ frequency range, depending on the number of silver ribbons connected to the central micrometric square mesh silver film. On the other hand, measured SE reaches 


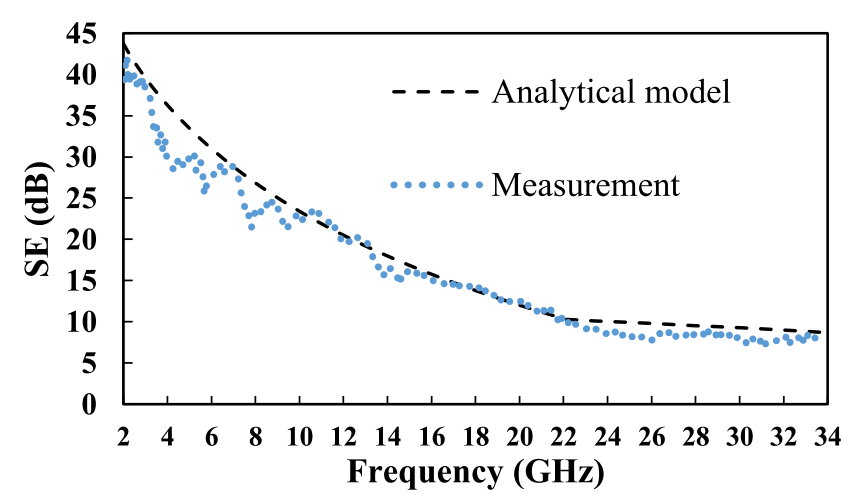

Fig. 10. Theoretical and measured SE versus frequency of the micrometric mesh silver sample with 132 silver ribbons.

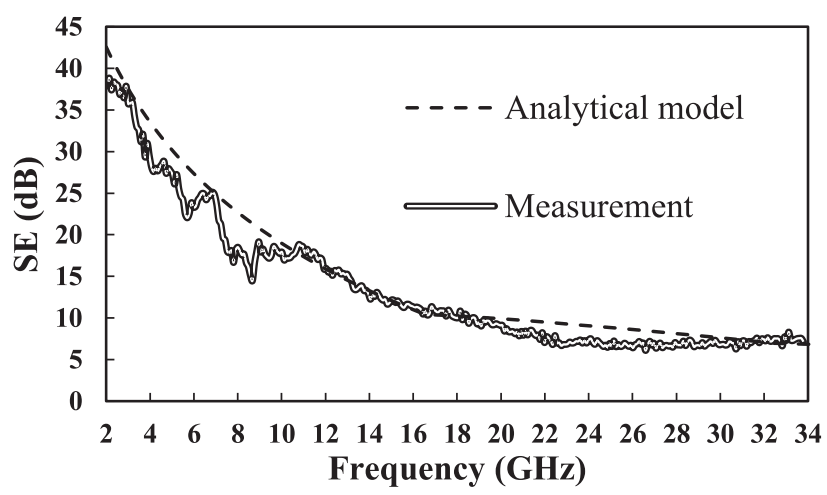

Fig. 11. Theoretical and measured SE versus frequency of the micrometric mesh silver sample with 92 silver ribbons.

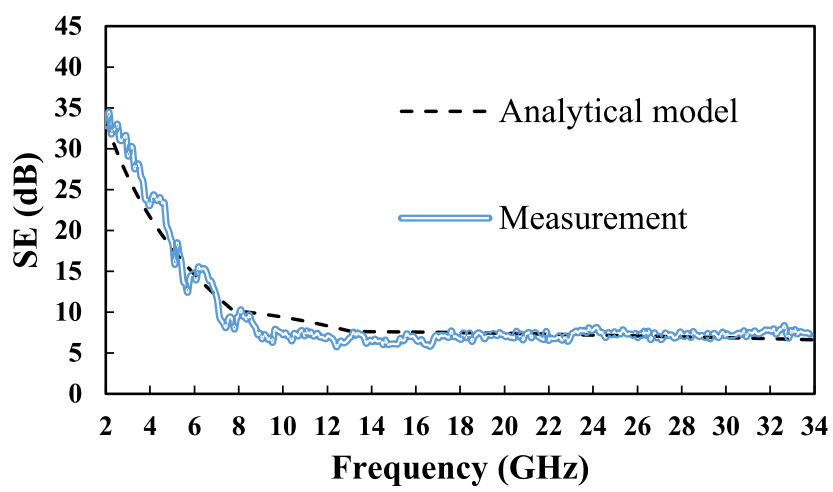

Fig. 12. Theoretical and measured SE versus frequency of the micrometric mesh silver sample with 44 silver ribbons.

a threshold value $(\sim 7 \mathrm{~dB})$ beyond this frequency range, as predicted by the model. Finally, Fig. 14 and Table III also highlight that SE threshold value is achieved at the smallest frequency with the sample involving the lowest number of silver ribbons $(\times 24)$. This article also demonstrates that the lack of control of only a few tens of conducting connections may yield to important SE variations in specific frequency spectrum of interest.

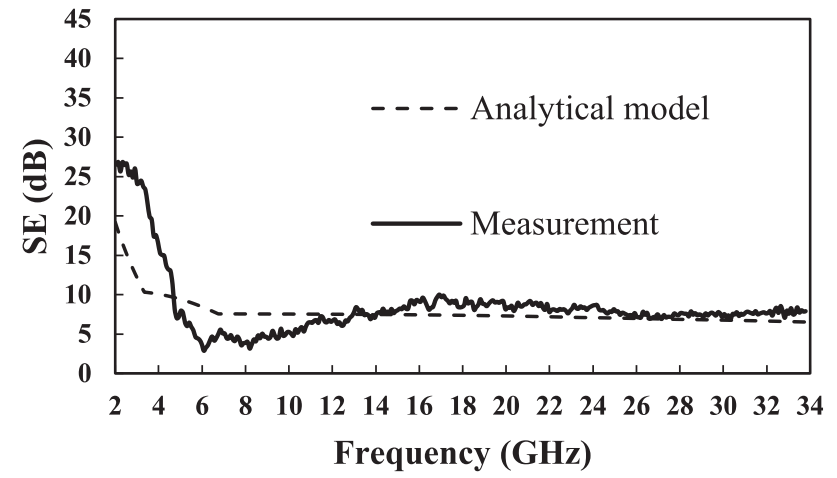

Fig. 13. Theoretical and measured SE versus frequency of the micrometric mesh silver sample with 24 silver ribbons.

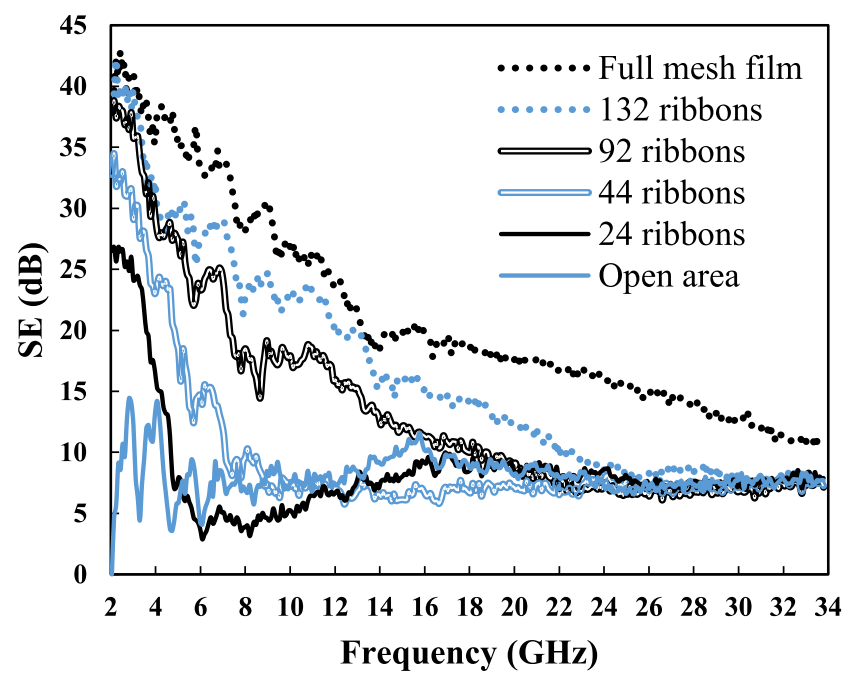

Fig. 14. Measured SE versus frequency of the micrometric mesh silver samples with various silver ribbons: $132,92,44$, and 24 . The two extreme cases of the study are also plotted: the full micrometric mesh silver sample and the central micrometric mesh silver sample surrounded by an open area.

TABLE III

FREQUENCIES AT VARIOUS SE LEVELS

\begin{tabular}{ccccc}
\hline \hline SE level & $25 \mathrm{~dB}$ & $20 \mathrm{~dB}$ & $15 \mathrm{~dB}$ & $10 \mathrm{~dB}$ \\
\hline Full mesh & $11.2 \mathrm{GHz}$ & $13.4 \mathrm{GHz}$ & $26.0 \mathrm{GHz}$ & $/$ \\
132 ribbons & $7.4 \mathrm{GHz}$ & $12.0 \mathrm{GHz}$ & $16.4 \mathrm{GHz}$ & $21.7 \mathrm{GHz}$ \\
92 ribbons & $5.4 \mathrm{GHz}$ & $7.3 \mathrm{GHz}$ & $13.0 \mathrm{GHz}$ & $18.0 \mathrm{GHz}$ \\
44 ribbons & $3.7 \mathrm{GHz}$ & $4.8 \mathrm{GHz}$ & $6.5 \mathrm{GHz}$ & $7.1 \mathrm{GHz}$ \\
24 ribbons & $2.6 \mathrm{GHz}$ & $3.6 \mathrm{GHz}$ & $4.2 \mathrm{GHz}$ & $4.7 \mathrm{GHz}$ \\
\hline \hline
\end{tabular}

\section{CONCLUSION}

The design, fabrication, and characterization of optically transparent electromagnetic shielding screens to protect against HIRF aggressions have been presented. The proposed solution is based, on the one hand on a micrometric mesh metal film with set mesh parameters, namely the pitch $g$ and the strip width $2 a$, to achieve the required optical transparency, and on the other hand on a controlled number of metal ribbons connecting 
the micrometer mesh metal film to its direct peripheral area. These metal ribbon connections allow for the modulation of SE performance according to different requirements. For this, a theoretical and approximate SE model has been specifically developed and compared with the experimental SE results. The strong agreement between theory and measurements thereby validates the study. Furthermore, the present solution based on a single micrometric mesh metal pattern controlling the SE level and frequency bandwidth of the shielding screens would greatly help its industrial implementation.

Moreover, the theoretical model introduced in this article was not confronted with numerical simulations of Maxwell equations in 3-D. Such simulations are challenging, as they require a very fine geometrical meshing of the micrometric mesh film and its peripheral ribbons. The substitution of the micrometric mesh film by an equivalent surface impedance boundary condition is a possible way to overcome this issue. This equivalent surface impedance boundary condition must be valid over a wide frequency range. This may be a possible perspective for this article.

\section{REFERENCES}

[1] Federal Aviation Administration, U.S. Department of Transportation, "Advisory circular 20-158A: The certification of aircraft electrical and electronic systems for operation in the high-intensity radiated fields (HIRF) environment," AC no.: 20-158A, May 2014.

[2] F. Colombel, X. Castel, M. Himdi, G. Legeay, S. Vigneron, and E. Motta Cruz, "Ultrathin metal layer, ITO film and ITO/Cu/ITO multilayer towards transparent antenna," IET Sci. Meas. Technol., vol. 3, no. 3, pp. 229-234, May 2009.

[3] Y.-J. Choi, S. C. Gong, D. C. Johnson, S. Golledge, G. Y. Yeom, and H.-H. Park, "Characteristics of the electromagnetic interference shielding effectiveness of $\mathrm{Al}$-doped $\mathrm{ZnO}$ thin films deposited by atomic layer deposition," Appl. Surf. Sci., vol. 269, pp. 92-97, Mar. 2013.

[4] D. S. Ghosh, L. Martinez, S. Giurgola, P. Vergani, and V. Pruneri, "Widely transparent electrodes based on ultrathin metals," Opt. Lett., vol. 34, no. 3, pp. 325-327, Feb. 2009.

[5] S. Rea, D. Linton, E. Orr, and J. McConnell, "New method for efficient HIRF attenuation in aircraft cooling ducts," in Proc. 22nd Digit. Avion. Syst. Conf., Indianapolis, IN, USA, Oct. 2003, pp. 1.B.4-1-1.B.4-8.

[6] Y. Corredores, P. Besnier, X. Castel, J. Sol, C. Dupeyrat, and P. Foutrel, "Adjustment of shielding effectiveness, optical transmission, and sheet resistance of conducting films deposited on glass substrates," IEEE Trans. Electromagn. Compat., vol. 59, no. 4, pp. 1070-1078, Aug. 2017.

[7] J. P. Kosiarski, "Consideration of mechanical and physical parameters on effective EMI shielding," in Proc. IEEE Int. Symp. Electromagn. Compat., Tokyo, Japan, Oct. 1984, pp. 524-527.

[8] E. Nakauchi and G. Fenical, "Contact surface resistivity: its effect on shielding effectiveness," in Proc. IEEE Int. Symp. Electromagn. Compat., Silicon Valley, CA, USA, Aug. 2004, pp. 838-840.

[9] G. Kunkel, "Transfer impedance testing of EMI gasketed joints to $10 \mathrm{GHz}$ for cost effective design," in Proc. IEEE Int. Symp. Electromagn. Compat., Pittsburgh, PA, USA, Aug. 2012 pp. 70-73.

[10] A. A. Eddib and D. D. L. Chung, "The importance of the electrical contact between specimen and testing fixture in evaluating the electromagnetic interference shielding effectiveness of carbon materials," Carbon, vol. 117, pp. 427-436, Jun. 2017.

[11] V. Gobin, and G. Labaune, "Calcul et mesure de l'efficacité de blindage des matériaux composites," Ann. Télécommun., vol. 43, no. 11/12, pp. 686694, Nov. 1988

[12] J. Catrysse, T. Grenson, F. Vanhee, D. Pissoort, and C. Brull, "The importance of continuous and homogeneous gasket conductivity up to $40 \mathrm{GHz}$," in Proc. IEEE Int. Symp. Electromagn. Compat., Brugge, Belgium, Sep. 2013 , pp. $536-540$.

[13] D. A. Hill, M. T. Ma, A. R. Ondrejka, B. F. Riddle, M. L. Crawford, and R. T. Johnk, "Aperture excitation of electrically large, lossy cavities," IEEE Trans. Electromagn. Compat., vol. 36, no. 3, pp. 169-178, Aug. 1994.
[14] H. A. Bethe, "Theory of diffraction by small holes," Phys. Rev., vol. 66, no. $7 / 8$, pp. $163-182$, Oct. 1944.

[15] K. S. H. Lee, "EMP interaction: principles, techniques and reference data: a handbook of technology from the EMP interaction notes," in Hemisphere Publishing Corporation, 1st ed. New York, NY, USA: Taylor \& Francis, 1986, pp. 1-744

[16] R. E. Collin, "Excitation of waveguides and cavities," in Field Theory of Guided Waves, 2nd ed. Hoboken, NJ, USA: Wiley, 1991, pp. 471-546.

[17] S. Celozzi, A. Araneo, and G. Lovat, "Apertures in planar metal screen," in Electromagnetic Shielding. Hoboken, NJ, USA: Wiley, 2008, pp. 144-163.

[18] N. A. McDonald, "Polynomial approximations for the electric polarizabilities of some small apertures," IEEE Trans. Microw. Theory Techn., vol. 33, no. 11 , pp. 1146-1149, Nov. 1985.

[19] N. A. McDonald, "Polynomial approximations for the transverse magnetic polarizabilities of some small apertures," IEEE Trans. Microw. Theory Techn., vol. 35, no. 1, pp. 20-23, Jan. 1987.

[20] M. Li, J. L. Drewniak, T. H. Hubbing, R. E. DuBroff, and T. P. VanDoren, "Slot and aperture coupling for airflow aperture arrays in shielding enclosure designs" in Proc. IEEE Int. Symp. Electromagn. Compat., Seattle, WA, USA, Aug. 1999 , pp. 35-39

[21] D. L. Jaggard, "Transmission through one or more small apertures of arbitrary shape," AFWL Interact. Note 323, Sep. 1977.

[22] F. Olyslager and D. De Zutter, "Skin effect," in Wiley Encyclopedia of Electrical and Electronics Engineering. Hoboken, NJ, USA: Wiley, 1999, vol. 19, pp. 1-6.

[23] W. Haynes, "Properties of solids," in CRC Handbook Chemistry Physics. Boca Raton, FL, USA: CRC Press, 2017, pp. 12-45.

[24] C. L. Holloway, D. A. Hill, J. Ladbury, G. Koepke, and R. Garzia, "Shielding effectiveness measurements of materials using nested reverberation chambers," IEEE Trans. Electromagn. Compat., vol. 45, no. 2, pp. 350-356, May 2003.

[25] A. Martin, O. Lafond, M. Himdi, and X. Castel, "Improvement of $60 \mathrm{GHz}$ transparent patch antenna array performance through specific double-sided micrometric mesh metal technology," IEEE Access, vol. 7, no. 1, pp. 22562262, Jan. 2019. 